\title{
Nondegenerate Four-Wave Mixing in a Homogeneously Broadened Two-Level System with Saturating Pump Waves
}

\author{
JOSEPH NILSEN AND AMNON YARIV, FELLOW, IEEE
}

\begin{abstract}
The formulation of phase conjugation via nondegenerate four-wave mixing in a homogeneously broadened resonant medium is generalized to include saturating pump waves. An ultrahigh- $Q$ optical filter is predicted, showing both gain and a bandwidth less than the homogeneous linewidth of the atom. The dependence of the filter function on the ac Stark effect and on the use of asymmetric pump wave intensities is discussed. In addition, several new phenomena are shown which manifest themselves as a dip in the degenerate reflectivity at line center.
\end{abstract}

\section{INTRODUCTION}

$\mathrm{R}^{\mathrm{E}}$ ECENT authors have discussed the theoretical solutions to phase conjugation via four-wave mixing in resonant systems [1]-[8]. The present authors first used third-order perturbation theory to study nondegenerate four-wave mixing in both homogeneously broadened [1] and inhomogeneously broadened [2], [3] two-level systems. These solutions showed how an ultrahigh- $Q$ optical filter could be constructed using phase conjugation. This has recently been verified experimentally using sodium as the nonlinear medium [9], [10]. Saturating pump waves have been included in the solutions of several authors for both degenerate [4], [5], [8] and nondegenerate [6], [7] four-wave mixing. In the degenerate case, our solutions predict two new phenomena which have not been previously recognized. One phenomenon is due to the relative rates at which the nonlinear susceptibility and the linear absorption saturate as a function of frequency. The second phenomenon is a result of the frequency dependence of the nonlinear susceptibility in the presence of asymmetric pump waves. In the nondegenerate case, we show how a narrow bandwidth optical filter with both gain and a linewidth less than the homogeneous linewidth of the system can be constructed. The important effects of asymmetric pump wave intensities on the efficiency and frequency response of the phase conjugate signal are also shown for the first time.

Manuscript received March 18, 1982; revised June 3, 1982. This work was supported by the U.S. Army Office, Durham, NC. The work of $J$. Nilsen was supported by the Fannie and John Hertz Foundation. J. Nilsen was a Fannie and John Hertz Foundation Graduate Fellow.

A. Yariv is with the Department of Electrical Engineering, California Institute of Technology, Pasadena, CA 91125.

J. Nilsen was with the Department of Electrical Engineering, California Institute of Technology, Pasadena, CA 91125 . He is now with the Physics Department, Lawrence Livermore National Laboratory, Livermore, CA 94550.

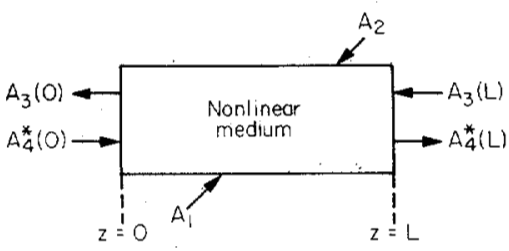

Fig. 1. Geometry for nondegenerate four-wave mixing (assuming nondepleting pump waves).

\section{Solving the Density Matrix Equations}

The mixing involves two intense counterpropagating pump waves $E_{1}$ and $E_{2}$ of the same frequency $\omega$ and two weak counterpropagating waves $E_{3}$ and $E_{4}$ with frequencies $\omega_{3}$ and $\omega_{4}$. The geometry of Yariv and Pepper [11] shown in Fig. 1 is used.

The fields are taken as plane waves:

$$
E_{i}\left(r_{i}, t\right)=\frac{1}{2} A_{i}\left(r_{i}\right) \exp \left[i\left(\omega_{i} t-\boldsymbol{k}_{i} \cdot \boldsymbol{r}\right)\right]+\text { c.c. }
$$

where $\boldsymbol{r}_{i}$ is the distance along $\boldsymbol{k}_{\boldsymbol{i}}$. We have

$$
\boldsymbol{k}_{1}+\boldsymbol{k}_{2}=0, \quad \omega_{3}+\omega_{4}=2 \omega \text {. }
$$

The resonant medium is modeled as a two-level system which is characterized by a dipole moment $\mu$, an enèrgy splitting $\hbar \omega_{0}$, and longitudinal and transverse relaxation times $T_{1}$ and $T_{2}$, respectively [12]. The two-level system is assumed to consist of an ensemble of stationary atoms. The density matrix equations are then solved to all orders in the amplitudes of the pump waves and to first order in the amplitudes of the weak signal fields. The induced polarizations at $\omega_{3}$ and $\omega_{4}$ are given by

$$
\begin{aligned}
P\left(\omega_{3}=\right. & \left.2 \omega-\omega_{4}, \boldsymbol{k}_{1} \cdot \boldsymbol{r}\right)=\frac{c}{4 \pi \omega_{3}} \\
& \cdot\left\{-i \alpha_{3}\left(\boldsymbol{k}_{1} \cdot \boldsymbol{r}\right) A_{3}+\kappa_{3}^{*}\left(\boldsymbol{k}_{1} \cdot \boldsymbol{r}\right) A_{4}^{*} \exp [i(\Delta k) z]\right\} \\
& \times \exp \left[i\left(\omega_{3} t-\boldsymbol{k}_{3} \cdot \boldsymbol{r}\right)\right] \\
P\left(\omega_{4}=\right. & \left.2 \omega-\omega_{3}, \boldsymbol{k}_{1} \cdot \boldsymbol{r}\right)=\frac{c}{4 \pi \omega_{4}} \\
& \cdot\left\{-i \alpha_{4}\left(\boldsymbol{k}_{1} \cdot \boldsymbol{r}\right) A_{4}+\kappa_{4}^{*}\left(\boldsymbol{k}_{1} \cdot \boldsymbol{r}\right) A_{3}^{*} \exp [i(\Delta k) z]\right\} \\
& \times \exp \left[i\left(\omega_{4} t-\boldsymbol{k}_{4} \cdot \boldsymbol{r}\right)\right] .
\end{aligned}
$$


The coupling constants appearing in (3) are

$$
\begin{aligned}
& \alpha_{3}\left(k_{1} \cdot r\right)=\frac{-i \alpha_{0}}{(\delta-\nu-i)\left[1+\frac{A^{*} A}{E_{s}^{2}\left(1+\delta^{2}\right)}\right]} \\
& \times\left[1-\frac{\frac{A^{*} A(2-i \nu)}{2 E_{s}^{2}(1-i a \nu)(1-i \delta)[1+i(\delta-\nu)]}}{\left[1+\frac{A^{*} A(1-i \nu)}{E_{s}^{2}(1-i a \nu)[1+i(\delta-\nu)][1-i(\delta+\nu)]}\right]}\right] \\
& \alpha_{4}^{*}\left(\boldsymbol{k}_{1} \cdot \boldsymbol{r}\right)=\frac{i \alpha_{0}}{(\delta+\nu+i)\left[1+\frac{A^{*} A}{E_{s}^{2}\left(1+\delta^{2}\right)}\right]} \\
& \times\left[1-\frac{\frac{A^{*} A(2-i \nu)}{2 E_{s}^{2}(1-i a \nu)(1+i \delta)[1-i(\delta+\nu)]}}{A^{*} A(1-i \nu)}\right] \\
& \kappa_{3}^{*}\left(\boldsymbol{k}_{1} \cdot \boldsymbol{r}\right)=2 \alpha_{0}\left[\frac{[1-(i \nu) / 2]}{(\delta-i)(1-i a \nu)(\delta-\nu-i)(\delta+\nu+i)}\right] \cdot \frac{A_{1} A_{2}}{E_{s}^{2}} \cdot \frac{1}{S} \\
& \kappa_{4}\left(k_{1} \cdot r\right)=2 \alpha_{0}\left[\frac{[1-(i \nu) / 2]}{(\delta+i)(1-i a \nu)(\delta-\nu-i)(\delta+\nu+i)}\right] \cdot \frac{A_{1}^{*} A_{2}^{*}}{E_{s}^{2}} \cdot \frac{1}{S} \\
& S=\left[1+\frac{A^{*} A}{E_{s}^{2}\left(1+\delta^{2}\right)}\right] \\
& \times\left[1+\frac{A^{*} A(1-i \nu)}{E_{s}^{2}(1-i a \nu)[1-i(\delta+\nu)][1+i(\delta-\nu)]}\right]
\end{aligned}
$$

with

$$
A=A_{1} e^{-i k_{1} \cdot r}+A_{2} e^{-i k_{2} \cdot r}
$$

and where $\delta=\left(\omega-\omega_{0}\right) T_{2}$ is the normalized detuning of the pump frequency from line center, $\nu=\left(\omega_{4}-\omega\right) T_{2}$ is the normalized detuning of the signal frequency from the pump frequency, $a=T_{1} / T_{2}, E_{s}^{2}=\hbar^{2} / T_{1} T_{2} \mu^{2}$ is the line-center saturation intensity, $\alpha_{0}=4 \pi \mu^{2} \Delta N_{0} T_{2} k_{0} / 2 h$ is the line-center homogeneous-broadening absorption coefficient of the subject gas, $k_{0}$ is the magnitude of the wave number at frequency $\omega_{0}$, and $\Delta k=2\left(\omega_{4}-\omega\right) / c$.

The coupling coefficients have a dc spatial component as well as high-frequency spatial components due to the $A^{*} A$ term in each coupling coefficient. Only the dc component of the coupling coefficients is phase matched and, therefore, of any importance in the coupled mode equations. Defining

$$
\begin{array}{ll}
A_{1}=\left|A_{1}\right| e^{i \vartheta_{1}} & A_{2}=\left|A_{2}\right| e^{i \vartheta_{2}} \\
I_{1}=\frac{\left|A_{1}\right|^{2}}{E_{s}^{2}} & I_{2}=\frac{\left|A_{2}\right|^{2}}{E_{S}^{2}}
\end{array}
$$

we obtain

$$
\frac{A^{*} A}{E_{s}^{2}}=I_{1}+I_{2}+2 \sqrt{I_{1} I_{2}} \cos (x+\vartheta)
$$

with $x=2 \boldsymbol{k}_{1} \cdot \boldsymbol{r}$ and $\vartheta=\vartheta_{2}-\vartheta_{1}$. The $\boldsymbol{k}_{2}$ dependence in (7) has been eliminated by using (2).

We can now determine the dc spatial component of $\alpha_{i}$ and $\kappa_{i}$ by integrating over $x$ :

$$
\begin{aligned}
& \alpha_{i}=\frac{1}{2 \pi} \int_{0}^{2 \pi} \alpha_{i}(x) d x \\
& \kappa_{i}=\frac{1}{2 \pi} \int_{0}^{2 \pi} \kappa_{i}(x) d x .
\end{aligned}
$$

The values of the coupling constants are determined from (8) by numerical integration on the IBM $370 / 3032$ computer. Analytic expressions for these integrals have been given by several authors for degenerate four-wave mixing [4], [5] and nondegenerate four-wave mixing [6], [7] in the case of equal intensity pump waves. Our numerical results agree with the analytic expressions; however, our paper presents a new analy. sis of the solutions which yield several phenomena that have not previously been predicted. For pump waves of different intensity, Dunning and Steel [8] present an analytic solution for the degenerate case, but do not analyze the frequency dependence of the reflectivity. The frequency analysis yields the phenomenon that is predicted in this work. For the nondegenerate case, the solutions we present have not previously been reported. 


\section{Coupled Mode Equations}

The values of $\alpha_{i}$ and $\kappa_{i}$ calculated in (8) can now be used in the coupled mode equations

$$
\begin{aligned}
& \frac{d A_{3}}{d z}=\alpha_{3} A_{3}+i \kappa_{3}^{*} A_{4}^{*} \exp [i(\Delta k) z] \\
& \frac{d A_{4}^{*}}{d z}=-\alpha_{4}^{*} A_{4}^{*}+i \kappa_{4} A_{3} \exp [-i(\Delta k) z] .
\end{aligned}
$$

The solutions to the above equations describe the evolution of waves $A_{3}$ and $A_{4}^{*}$.

We will study the dependence of the reflected signal on the various parameters such as frequency, linear absorption, and pump intensity for the case of a single input wave $A_{4}^{*}(0)$ with $A_{3}(L)=0$. The reflectivity $R$ is defined as

$$
R \equiv\left|A_{3}(0)\right|^{2} /\left|A_{4}(0)\right|^{2}
$$

with its solution given by

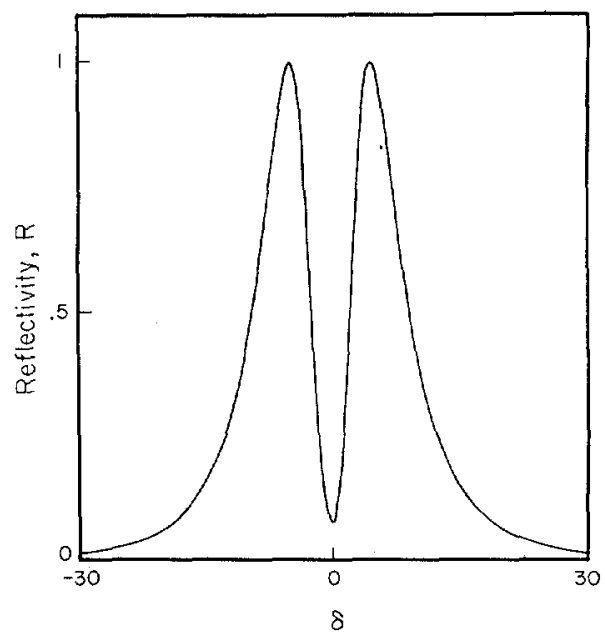

Fig. 2. Reflectivity versus the pump detuning $\delta$ for $\alpha_{0} L=60$ and $I=$ 100 . The curve is normalized to unity at its maxima.

$$
R=\left|\frac{\kappa_{3}^{*}\left\{1-\exp \left[i\left(S_{1}-S_{2}\right) L\right]\right\}}{i\left\{S_{1}-S_{2} \exp \left[i\left(S_{1}-S_{2}\right) L\right]\right\}+\alpha_{3}\left\{\exp \left[i\left(S_{1}-S_{2}\right) L\right]-1\right\}}\right|^{2}
$$

where

$$
\begin{aligned}
S_{1}= & (-i / 2)\left(\alpha_{3}-\alpha_{4}^{*}+i \Delta k\right)+(1 / 2)\left[4 \kappa_{3}^{*} \kappa_{4}-\left(\alpha_{3}+\alpha_{4}^{*}\right)^{2}\right. \\
& \left.+(\Delta k)^{2}+2 i \Delta k\left(\alpha_{3}+\alpha_{4}^{*}\right)\right]^{1 / 2} \\
S_{2}= & (-i / 2)\left(\alpha_{3}-\alpha_{4}^{*}+i \Delta k\right)-(1 / 2)\left[4 \kappa_{3}^{*} \kappa_{4}-\left(\alpha_{3}+\alpha_{4}^{*}\right)^{2}\right. \\
& \left.+(\Delta k)^{2}+2 i \Delta k\left(\alpha_{3}+\alpha_{4}^{*}\right)\right]^{1 / 2} .
\end{aligned}
$$

First we will investigate the degenerate case and discuss several new phenomena which are predicted by our solution for $R$. This will be followed by an analysis of the nondegenerate case and a discussion of a variety of interesting phenomena associated with it.

\section{Degenerate Four-Wave Mixing}

The first new phenomenon predicted is a dip in the degenerate $(\nu=0)$ reflectivity at $\delta=0$ when $R$ is plotted versus $\delta$. This is shown in Fig. 2 where $R$ is plotted versus $\delta$ for $I=100$, $T_{1} / T_{2}=1 / 2$, and $\alpha_{0} L=60$ with $I \equiv I_{1}=I_{2}$ for the case of equal intensity pump waves. The curve is normalized to unity at its maximum. As explained below, the magnitude of the dip increases as $\alpha_{0}$ increases.

To explain this phenomenon, recall that the reflectivity $R$ can be approximated [2] by

$$
R \approx\left|\kappa_{3} L\right|^{2}\left(1-e^{-2 \alpha_{R} L}\right)^{2} / 4 \alpha_{R}^{2} L^{2}
$$

with $2 \alpha_{R}=\operatorname{Re}\left(\alpha_{3}+\alpha_{4}^{*}\right)$ for $R<1$. In the degenerate case, $\kappa_{3}=\kappa_{4} \equiv \kappa$. For small $\alpha_{R} L<1, R \approx|\kappa L|^{2}$, but for large $\alpha_{R} L>1, R \approx\left|\kappa / 2 \alpha_{R}\right|^{2}$. Now $|\kappa|$ and $\alpha_{R}$ both peak at $\delta=0$, but suppose that $\alpha_{R}$ is a more sharply peaked function than $|\kappa|$. If at $\delta=0, \alpha_{R} L$ is larger than unity, then $R \approx\left|\kappa / 2 \alpha_{R}\right|^{2}$. As $|\delta|$ is increased, the reflectivity will initially increase because $\alpha_{R}$ will decrease faster than $|\kappa|$. Eventually, $|\delta|$ will increase to the point that $\alpha_{R} L<1$, implying that $R \approx|\kappa L|^{2}$, and $R$ will then decrease with increasing $|\delta|$. The net result will be a dip at $\delta=0$ whose magnitude depends both on the relative dependences of $\kappa$ and $\alpha_{R}$ on $\delta$ and the value of $\alpha_{R} L$ at $\delta=0$. The important criterion for observing a dip is that $\alpha_{R}$ be more sharply peaked than $\kappa$ around $\delta=0$.

In Fig. 3, $|\kappa|$ and $\alpha_{R}$ are both plotted versus $\delta$ for $I=100$. The curves are both normalized to unity at $\delta=0$. In the saturated regime, $\alpha_{R}$ is more sharply peaked than $|\kappa|$, and the differences in the responses increases with $I$. Therefore, we would expect to see the dips shown in Fig. 2.

The second new phenomenon predicted is a dip in the reflectivity at $\delta=0$ due to an asymmetry in the pump wave intensities. This is shown in Fig. 4 where the reflectivity $R$ is plotted versus $\delta$ for various values of $I_{1}$. The product $I_{1} \cdot I_{2}=10^{4}$ is kept constant and $\alpha_{0} L=1$. The curves are normalized to unity for $I_{1}=100$ and $\delta=0$. As the asymmetry in the amplitudes of the pump waves increases, we observe a decrease in the amplitude of $R$ and a dip at $\delta=0$. We have chosen $\alpha_{0} L=1$ to distinguish this phenomenon from the previous one by ensuring that we are in the regime where $R \approx|\kappa L|^{2}$.

\section{Nondegenerate Four-Wave Mixing}

Having considered the degenerate case, we will now study the nondegenerate case, $\nu \neq 0$. First we demonstrate that a narrow bandwidth optical filter can be constructed using nondegenerate four-wave mixing in the saturated regime. Using $\alpha_{0} L=65, T_{1} / T_{2}=1 / 2$, and $\delta=8$, Fig. 5 shows the reflectivity plotted versus $\nu$ for $I=36$. For this choice of $\delta$, the value of $I$ used corresponds to a maximum value of the reflectivity at $\nu=0$. Fig. 5 demonstrates that this process can yield an optical filter whose bandwidth depends on the inverse of the lifetime $T_{2}$, but which can be narrowed even more by operating in the gain regime and taking advantage of the exponential dependence of $R$ on $\kappa_{i}$. If we choose $T_{2}=32 \mathrm{~ns}$, the FWHM bandwidth of the filter is $4 \mathrm{MHz}$. By comparison, the homogeneous linewidth of the transition is $10 \mathrm{MHz}$ for this value of $T_{2}$. When operated in the unsaturated regime [1], the filter's bandwidth would then be limited by the homogenebus linewidth. However, with the use of saturating pump waves to achieve gain, 


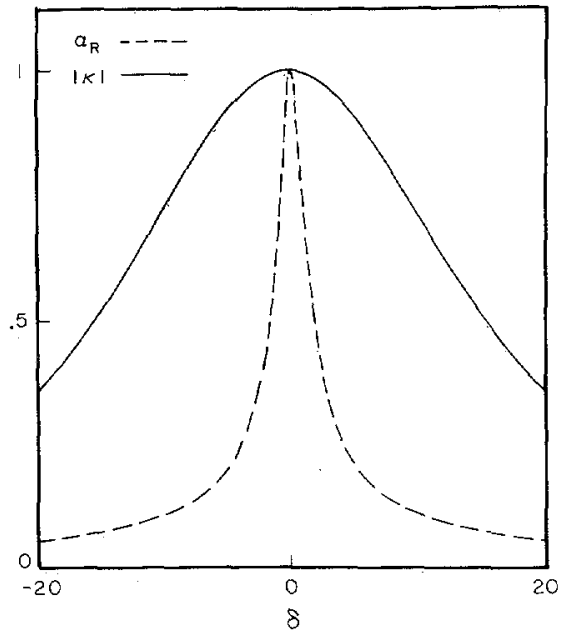

Fig. 3. $\alpha_{R}$ and $|\kappa|$ plotted verus the pump detuning $\delta$ for $I=100$. Both curves are normalized to unity at $\delta=0$.

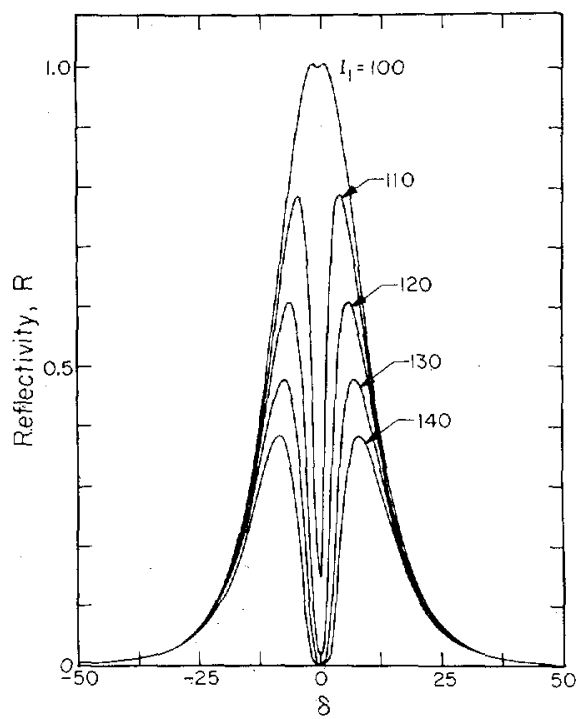

Fig. 4. Reflectivity versus the pump detuning $\delta$ for several values of $I_{1}$ with the product $I_{1} \cdot I_{2}=10^{4}$. The curves are normalized to unity at $\delta=0$ and $I_{1}=100$.

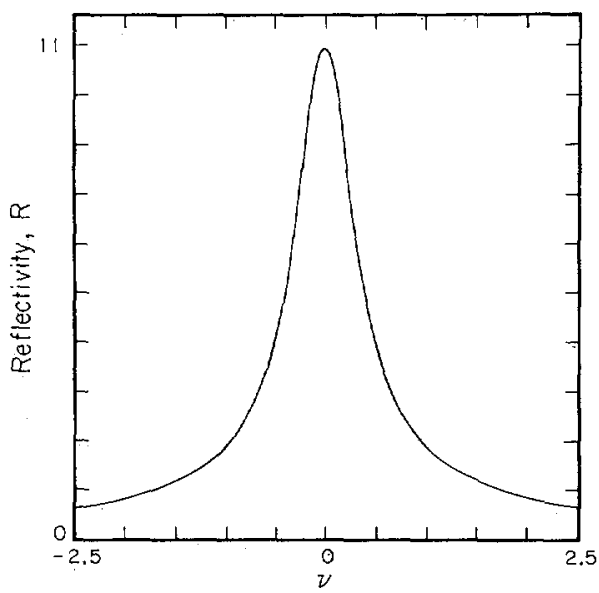

Fig. 5. Reflectivity versus the signal detuning $\nu$ for $I=36$ with $\delta=8$ and $\alpha_{0} L=65$. the filter has a subhomogeneous bandwidth as shown in Fig. 5 . Having demonstrated the filter concept, we spend the remainder of the section discussing the ac Stark effect.

To simplify the discussion, we can define a normalized Rabi frequency

$$
\nu_{R}=\Omega_{g} T_{2}=\sqrt{\delta^{2}+\left|\frac{\mu A T_{2}}{\hbar}\right|^{2}} .
$$

Assume that the strong pump waves at frequency $\omega$ cause the ac Stark effect. We would then expect a resonance in the reflectivity $R$ when $\nu=0, \pm \nu_{R}$ as well as the usual resonances at $\pm \delta$. The case $\nu=0$ corresponds to the signal waves and pump waves all being resonant with one transition, while the cases $\nu= \pm \nu_{R}$ correspond to the situation in which each of the four waves is resonant with a different transition. This is better illustrated in Fig. 6 where the energy levels of the twolevel system in the presence of strong pump waves are shown. The strong pump waves cause a splitting $\hbar \Omega_{g}$ in both the ground state and the excited state. This figure shows the case $\omega_{4}=\omega+\Omega_{g}, \omega_{3}=\omega-\Omega_{g}$, which implies a resonance at $\nu=\nu_{R}$.

To relate the Rabi frequency to the pump wave amplitude in the case of four-wave mixing, we consider equal amplitude pump waves. From (5) and the definition of $E_{s}^{2}$, we have

$$
\left|\frac{\mu A T_{2}}{\hbar}\right|^{2}=\frac{2 I}{a}[1+\cos (x+\vartheta)] .
$$

Now, the most probable value of a cosine is at its maximum or minimum so we can expect the right side of (15) to have either 0 or $4 I / a$ as its most probable value. Since 0 results in no Stark splitting, we expect the Stark splitting to be such that

$$
\nu_{R}=\sqrt{\delta^{2}+\frac{4 I}{a}} .
$$

Having described the Stark effect, we now consider the calculated reflectivity and compare it to our model.

Considering the case of equal intensity pump waves first, we chose the parameters to be $\alpha_{0} L=1, I=100$, and $T_{1} / T_{2}=1 / 2$. Fig. 7 shows the reflectivity $R$ versus $\nu$ for $\delta=8$. In this plot, we observe a peak at $\nu=0$ and $\pm \delta$ as well as the sidebands due to the ac Stark effect at $\nu= \pm 29.12$. The basic structure has been observed in recent experiments using sodium as the nonlinear medium [10]. The value calculated from (16) for $\nu_{R}=$ 29.39 agrees very well with the observed value. We have also compared the position of the side peaks to $\nu_{R}$ as a function of $\delta$ and $T_{1} / T_{2}$ and also obtain good agreement. A similar discussion has been presented by Harter and Boyd [7] for the case of equal amplitude pump waves. However, they do not consider the important effect an asymmetry in the pump wave amplitudes has on the reflectivity, an effect which manifests itself in the new phenomenon presented in this section.

For the case of pump waves of different intensity, Fig. 8 shows $R$ plotted versus $v$ for $I_{1}=400, I_{2}=25, I_{1} \cdot I_{2}=10^{4}$, $\delta=0$, and the other parameters unchanged from the previous figure. As $I_{1}$ increases, the amplitude of the central peak 


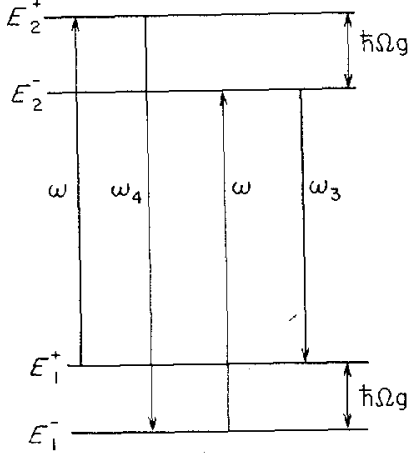

Fig. 6. Energy levels of the two-level system in the presence of strong pump waves.

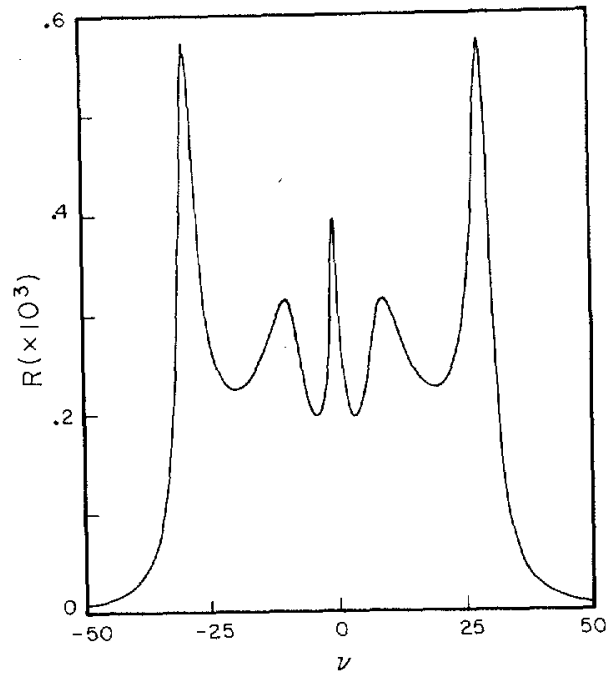

Fig. 7. Reflectivity versus signal detuning $\nu$ for $\delta=8$ and $I=100$.

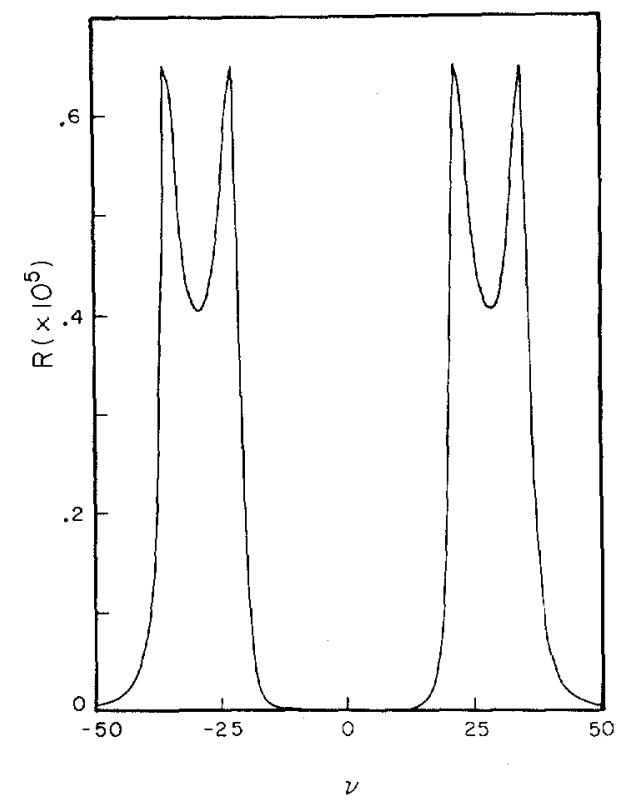

Fig. 8. Reflectivity versus signal detuning $v$ for $I_{1}=400$ and $I_{2}=25$ with $\delta=0$.

diminishes. This peak then splits into two peaks whose separation increases with $I_{1}$.
We can understand this double-peaked structure in each sideband by recalling (7), which gives the standing wave pattern for pump wave intensity. In the case of asymmetric pump wave amplitudes, this equation results in a modification of (15) to

$$
\left|\frac{\mu A T_{2}}{\hbar}\right|^{2}=\frac{1}{a}\left[I_{1}+I_{2}+2 \sqrt{I_{1} I_{2}} \cos (x+\vartheta)\right] .
$$

Again using the fact that the cosine function has \pm 1 as its most probable value, we expect that the normalized Rabi frequency $v_{R}$ is given by

$$
\nu_{R}^{2}=\frac{1}{a}\left[I_{1}+I_{2} \pm 2 \sqrt{I_{1} I_{2}}\right]+\delta^{2} .
$$

Defining $v_{R}$ to be the positive root, the resonances occur at $\nu= \pm \nu_{R}$, with $\nu_{R}$ being double valued. Taking the case $I_{1}=$ 400 and $I_{2}=25$, this gives $\nu_{R}=21.21$ and 35.36, which agrees very well with the position of the sidebands which are observed in Fig. 8 at $\nu=22.23$ and 34.72. The observed peaks coincide quite closely with the resonances expected from the calculated values of $\nu_{R}$.

\section{CONCLUSION}

This paper has presented a general solution for nondegenerate four-wave mixing in a homogeneously broadened two-level system including the effect of saturating pump waves. In the degenerate case, the analysis predicts several new features in the frequency dependence of the reflectivity. The first is a dip in the reflectivity at line center due to the relative powerbroadened linewidths of the linear absorption coefficient and the nonlinear (third-order) susceptibility. The second interesting phenomenon also manifested itself as a dip in the reflectivity at line center. The dip results from the dependence of the nonlinear coupling coefficient $k$ on the asymmetry in the pump wave amplitudes.

In the nondegenerate case, we presented the dependence of the reflection coefficient on the signal detuning $\nu$. We demonstrated a narrow bandwidth optical filter which has an efficiency greater than unity and a bandwidth less than the homogeneous linewidth. We considered in detail the ac Stark effect and how it generates sidebands in the filter response whose location depends on the pump wave intensities. This could be used to construct a tunable filter which utilizes this effect to control the frequency of the central bandpass of the filter. As a spectroscopic tool, we could use the location of the Rabi sidebands as a measure of the dipole moment of the atom.

\section{REFERENCES}

[1] J. Nilsen and A. Yariv, "Nearly degenerate four-wave mixing applied to optical filters," Appl. Opt., vol. 18, pp. 143-145, 1979.

[2] — "Nondegenerate four-wave mixing in a Doppler-broadened resonant medium," J. Opt. Soc. Amer., vol. 71, pp. 180-183, 1981.

[3] - "A tunable narrowband optical filter via phase conjugation by nondegenerate four-wave mixing in a Doppler-broadened resonant medium," Opt. Commun., vol. 39, pp. 199-204, 1981.

[4] R. L. Abrams and R. C. Lind, "Degenerate four-wave mixing in 
absorbing media," Opt. Lett., vol. 2, pp. 94-96, 1978.

[5] - "Degenerate four-wave mixing in absorbing media: Errata," Opt. Lett., vol. 3, p. 205, 1978.

[6] F. Tao-Yi and M. Sargent III, "Effects of signal detuning on phase conjugation," Opt. Lett., vol. 4, pp. 366-368, 1979.

[7] D. J. Harter and R. W. Boyd, "Nearly degenerate four-wave mixing enhanced by the ac Stark effect," IEEE J. Quantum Electron., vol. QE-16, pp. 1126-1131, 1980.

[8] G. J. Dunning and D. G. Steel, "Effects of unequal pump intensity in resonantly enhanced degenerate four-wave mixing," IEEE J. Quantum Electron., vol. QE-18, pp. 3-5, 1982.

[9] J. Nilsen, N. S. Gluck, and A. Yariv, "Narrowband optical filter via phase conjugation by nondegenerate four-wave mixing in sodium vapor," Opt. Lett., vol. 6, pp. 380 382, 1981.

[10] D. G. Steel and R. C. Lind, "Multiresonant behavior in nearly degenerate four-wave mixing: The ac Stark effect," Opt. Lett., vol. 6, pp. $587-589,1981$.

[11] A. Yariv and D. M. Pepper, "Amplified reflection, phase conjugation, and oscillation in degenerate four-wave mixing," Opt. Lett., vol, 1, pp. 16-18, 1977.

[12] A. Yariv, Quantum Electronics. New York: Wiley, 1975, pp. 149-155.

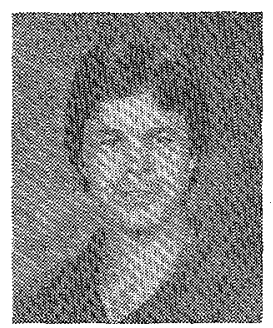

Joseph Nilsen was born in Brooklyn, NY, on December 16, 1955. He received the B.S. degree in engineering physics from Cornell University, Ithaca, NY, in 1977, and the M.S. and Ph.D. degrees in physics from the California Institute of Technology, Pasadena, in 1979 and 1982, respectively. At Caltech, he was a Fannie and John Hertz Foundation graduate fellow. His doctoral thesis included the first experimental demonstration of an ultrahigh- $Q$ optical filter utilizing the phase conjugation process.

Since 1977 he has been a Physicist in the Special Studies Group of the Physics Department at Lawrence Livermore National Laboratory, Livermore, CA, where he has carried out research on various aspects of optical communications and atomic physics. His present research interests include the development of short wavelength lasers and the study of nonequilibrium radiation phenomena.

Dr. Nilsen is a member of the Optical Society of America.

Amnon Yariv (S'56-M'59-F'70), for a photograph and biography, see p. 337 of the March 1982 issue of this Journat.

\title{
Dispersion-Induced Instability in CW Laser Oscillators
}

\author{
MONICA L. MINDEN AND LEE W. CASPERSON, MEMBER, IEEE
}

\begin{abstract}
A CW-pumped laser with a short photon cavity lifetime may show an unstable output in the form of spontaneous pulsations or noise. In this paper, a model is developed which reconciles previous studies of unstable behavior in homogeneously and inhomogeneously broadened lasers. Specific regions of instability are predicted for xenon lasers, and it is concluded that some degree of inhomogeneous broadening makes the instability much more likely to be observed.
\end{abstract}

\section{INTRODUCTION}

I $T$ has been shown that strong dispersion about an oscillating laser mode can cause the mode to split into a number of submodes, each of which satisfies the same oscillation condition as the original mode [1]. Beating between the modes can result in unstable operation or the generation of spontaneous pulses and is, in a sense, a form of self-mode-locking. However, the repetition rate of the pulses is related to the cavity losses and to the fundamental lifetimes and linewidths of the laser medium rather than to the empty cavity mode spacing.

Manuscript received March 24, 1981; revised May 24, 1982.

M. L. Minden is with the School of Engineering and Applied Science, University of California, Los Angeles, CA 90024 and the Hughes Research Laboratory, Malibu, CA 90265.

L. W. Casperson is with the School of Engineering and Applied Science, University of California, Los Angeles, CA 90024.
The existence of such pulsations has been demonstrated in experiments with $3.51 \mu \mathrm{m}$ xenon lasers [2]-[4] and in direct numerical integration of the semiclassical laser equations [5]. Similar and perhaps related periodic pulsation effects have been observed in several other laser systems. In $\mathrm{CO}_{2}$, for example, spontaneous pulsations are well known, but they have been attributed to transverse mode beating [6] or to a saturable absorption mechanism [7]. Intense but aperiodic fluctuations have also been observed in high gain $3.39 \mu \mathrm{m}$ helium-neon lasers [3], [8].

Conditions under which semiclassical instabilities may occur have been given in many places, including [1], [9]-[13]. One general condition for instability that has been obtained in these analyses can be written approximately as

$$
t_{c}<\left(2 \pi \Delta v_{h}\right)^{-1}
$$

where $t_{c}$ is the photon lifetime within the cavity and $\Delta \nu_{h}$ is the homogeneous linewidth. Each of the studies, however, has incorporated certain simplifying assumptions which make further comparison of results somewhat difficult. Risken and Nummedal [10] explored self-pulsing in homogeneously broadened lasers using the assumption that the population relaxation phenomena can be characterized by a single decay rate. Yaku- 